\title{
Final Technical Report:
}

\section{High Resolution PET with 250 micrometer LSO Detectors and Adaptive Zoom DE-FG02-08ER64677}

\author{
Project Period: $\quad$ 09/15/2008 - 09/14/2011 \\ Pls: \\ Simon Cherry, Ph.D. and Jinyi Qi, Ph.D. \\ University of California, Davis
}

\section{Introduction:}

There have been impressive improvements in the performance of small-animal positron emission tomography (PET) systems since their first development in the mid 1990s, both in terms of spatial resolution and sensitivity, which have directly contributed to the increasing adoption of this technology for a wide range of biomedical applications. Nonetheless, current systems still are largely dominated by the size of the scintillator elements used in the detector. Our research predicts that developing scintillator arrays with an element size of $250 \mu \mathrm{m}$ or smaller will lead to an image resolution of $500 \mu \mathrm{m}$ when using ${ }^{18} \mathrm{~F}$ - or ${ }^{64} \mathrm{Cu}$-labeled radiotracers, giving a factor of 4-8 improvement in volumetric resolution over the highest resolution research systems currently in existence. This proposal had two main objectives:

(i) To develop and evaluate much higher resolution and efficiency scintillator arrays that can be used in the future as the basis for detectors in a small-animal PET scanner where the spatial resolution is dominated by decay and interaction physics rather than detector size.

(ii) To optimize one such high resolution, high sensitivity detector and adaptively integrate it into the existing microPET II small animal PET scanner as a "zoom-in" detector that provides higher spatial resolution and sensitivity in a limited region close to the detector face.

The knowledge gained from this project will provide valuable information for building future PET systems with a complete ring of very high-resolution detector arrays and also lay the foundations for utilizing high-resolution detectors in combination with existing PET systems for localized high-resolution imaging.

\section{OBJECTIVE 1: DEVELOPMENT/CHARACTERIZATION OF HIGHLY SEGMENTED SCINTILLATOR ARRAYS}

Aim: To develop and evaluate much higher resolution and efficiency scintillator arrays that can be used in the future as the basis for detectors in a small-animal PET scanner where the spatial resolution is dominated by decay and interaction physics rather than detector size.

In PET, the spatial resolution depends on several factors, among them being the crystal width of the scintillators used in the detector. The goal of this proposal was to study very highly segmented scintillator arrays, with crystal sizes significantly less than $1 \mathrm{~mm}$. We fabricated and studied lutetium oxyorthosilicate (LSO) scintillator arrays with individual pixels sizes of $0.7 \times 0.7$ $\times 20 \mathrm{~mm}^{3}, 0.5 \times 0.5 \times 20 \mathrm{~mm}^{3}$ and $0.22 \times 0.22 \times 20 \mathrm{~mm}^{3}$. The arrays with elements of sizes of $0.5 \mathrm{~mm}$ and less, are to our knowledge the most finely segmented scintillator arrays yet studied for PET, and still maintained high efficiency through the use of $20 \mathrm{~mm}$ deep crystals. The results for the 0.5 and $0.7-\mathrm{mm}$ element arrays have been described in detail in the publications 
by (St. James et al, 2009). We also developed tapered scintillator arrays to improve system sensitivity by reducing the gaps between modules for a small-diameter system. Monte Carlo simulations of the performance of scanners based on tapered scintillator arrays were reported in (St. James et al, 2010). This report concentrates therefore on the experimental results from the far more challenging $0.22-\mathrm{mm}$ element LSO array.

The LSO used in this $0.22-\mathrm{mm}$ detector array was grown specifically for our project by $\mathrm{Dr}$. Chuck Melcher (University of Tennessee), the world's foremost authority on LSO growth. It was doped in a manner to be optimized for light output, critical for being able to decode these tiny scintillator elements that have a length:width (aspect ratio) of 90:1. The detector is a continuous depth-ofinteraction (DOI) detector and is read out at both ends using $8 \times 8 \mathrm{~mm}^{2}$ position sensitive avalanche photodiodes (PSAPDs) developed by Radiation Monitoring Devices (Watertown, MA). A photograph of the array along with an image under a microscope is shown in Figure 1. In the image, the 63.5 micron Vikuiti ${ }^{\mathrm{TM}}$ enhanced specular reflector (3M, St. Paul, MN) is visible.

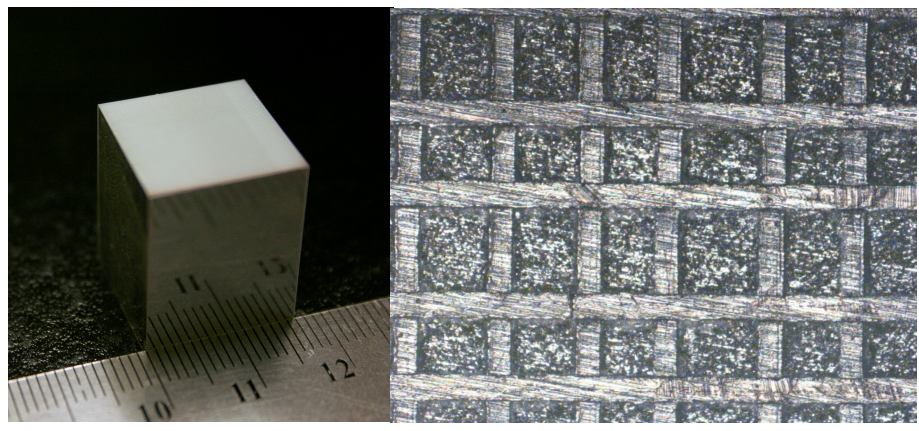

Figure 1: A photograph of the $0.22 \times 0.22 \times 20 \mathrm{~mm}^{3}$ LSO array (left). On the right is an image of the same array under a microscope.

The detectors were cooled to $-10^{\circ} \mathrm{C}$ to improve the signal to noise of the PSAPDs used to readout the arrays. Figure 2 shows a flood histogram obtained by irradiating the crystal array from the side (using electronic collimation to achieve a beam width localized in depth to $\sim 1.4$ $\mathrm{mm}$ ) at a depth of $5 \mathrm{~mm}$. The majority of the $0.22-\mathrm{mm}$ LSO elements can be resolved. Figure 3 shows the depth of interaction response on the detector, using the ratio of the two PSAPD outputs, and using a calibration curve to convert this to interaction depth. The average depth of interaction resolution of this detector is approximately $3 \mathrm{~mm}$, without correcting for the exciting beam width of $\sim 1.4 \mathrm{~mm}$.

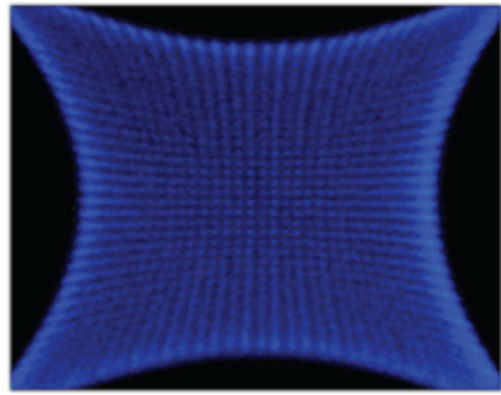

Figure 2: Flood histogram from $0.22 \times 0.22 \times 20 \mathrm{~mm}^{3}$ LSO array irradiated at a depth of $5 \mathrm{~mm}$. Pincushion distortion is a feature of the PSAPD readout and can be corrected.

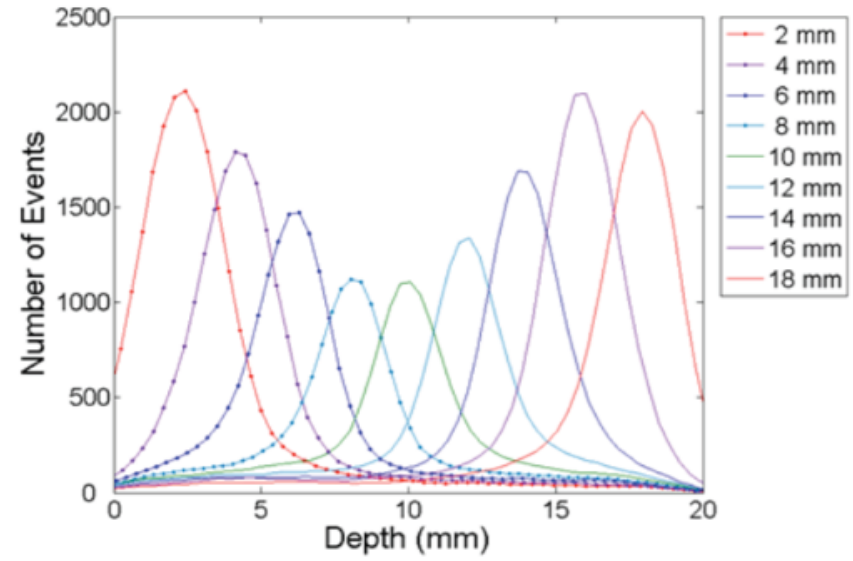

Figure 3: Histograms showing depth response of the $0.22-\mathrm{mm}$ element LSO detector for 9 different irradiation depths. From this data, the DOI resolution is estimated to be around $3 \mathrm{~mm}$ (not correcting for $1.4 \mathrm{~mm}$ estimated excitation beam width. 
To further characterize the performance of this detector the crystal response function was measured and compared to that of a $0.5 \times 0.5 \times 20 \mathrm{~mm}^{3}$ LSO PET detector. For both experiments, the crystal arrays were read out using $8 \mathrm{~mm} \times 8 \mathrm{~mm}$ PSAPDs and the detectors were cooled to $0{ }^{\circ} \mathrm{C}$.

To measure the crystal response function we designed a tungsten collimator $(2 \mathrm{~cm}$ thick $)$ that we mounted on a mechanical translation stage with accuracy of $2 \mu \mathrm{m}$. The tungsten was separated with $25 \mu \mathrm{m}$ shim tape and a ${ }^{22} \mathrm{Na}$ source was mounted behind the collimator. To measure the crystal response function, the collimator-source assembly was translated across the face of the detector. To minimize the contribution due to the intrinsic LSO background, an additional detector was used in coincidence with the evaluated detector. The collimating detector was a $2 \times 2 \times 2 \mathrm{~cm}^{3}$ LSO array coupled to a Hamamatsu M-64 photomultiplier tube (PMT). An illustration of the electronic setup is shown in Figure 4 and a photograph of the mechanical setup is shown in Figure 5.

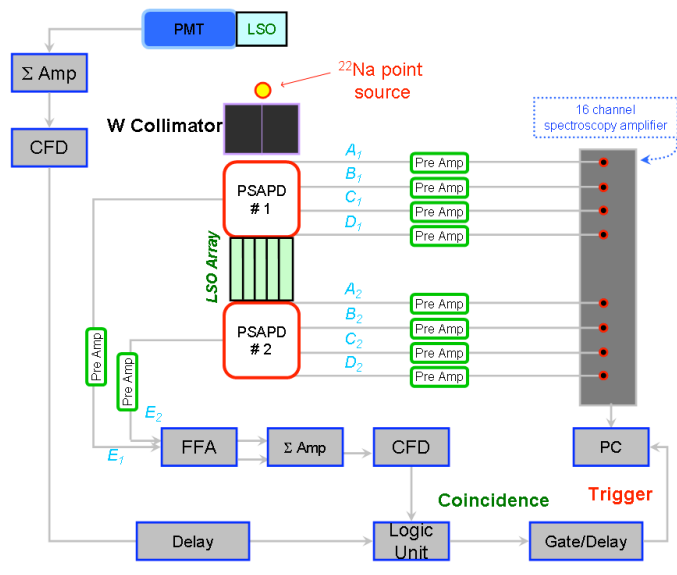

Figure 4: Illustration of the electronic setup for measuring the crystal response function.

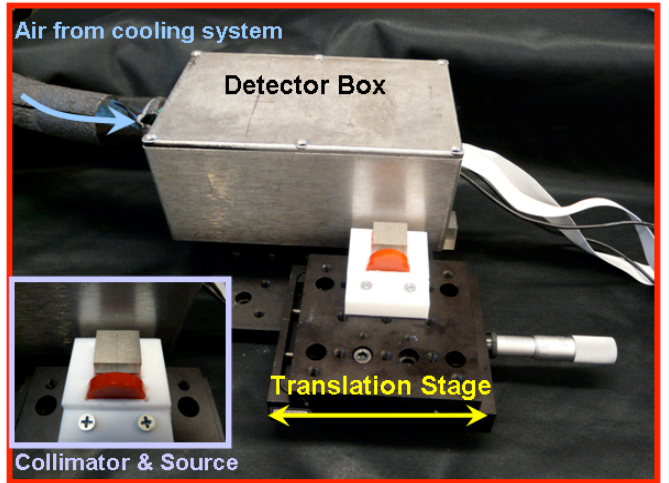

Figure 5: Photograph of the mechanical setup for measuring the crystal response function (without the collimating PMT).

Figure 6 shows flood histograms of the center of the $0.22 \times 0.22 \times 20 \mathrm{~mm}^{3}$ LSO PET detector when irradiated by the source-collimator assembly in two separate positions. In both figures, two lines of crystals are clearly visible and the individual crystals may be clearly distinguished. Figure 7 shows the normalized crystal response function for the $0.5 \times 0.5 \times 20 \mathrm{~mm}^{3}$ LSO array and for the $0.22 \times 0.22 \times 20 \mathrm{~mm}^{3}$ LSO array. The crystal response function for the $0.22 \times 0.22 \mathrm{x}$ $20 \mathrm{~mm}^{3}$ array shown is the summed crystal response function from four separate crystal rows (shifted by $0.29 \mathrm{~mm}$, the crystal pitch).

The crystal response function for the $0.22 \times 0.22 \times 20 \mathrm{~mm}^{3}$ LSO array is slightly better than that for the $0.5 \times 0.5 \times 20 \mathrm{~mm}^{3}$ LSO array, demonstrating that the resulting spatial resolution with such a detector would be better than for the $0.5 \times 0.5 \times 20 \mathrm{~mm}^{3}$ detector. The exact improvements on the effect on spatial resolution will be explored through Monte Carlo simulation (GATE) and characterization of the specific blurring functions that result in these crystal response functions. These experiments will be repeated to ensure (a) reproducibility and (b) better statistics. This is the first characterization of such a finely pixelated detector and also presents a new method by which single detectors may be evaluated. 


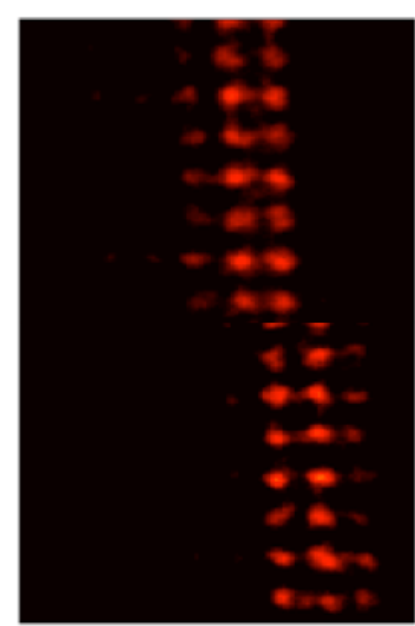

Figure 6: Two flood images of the 0.22 x $0.22 \times 20 \mathrm{~mm}^{3}$ LSO array irradiated with the collimator-source assembly in two separate positions.

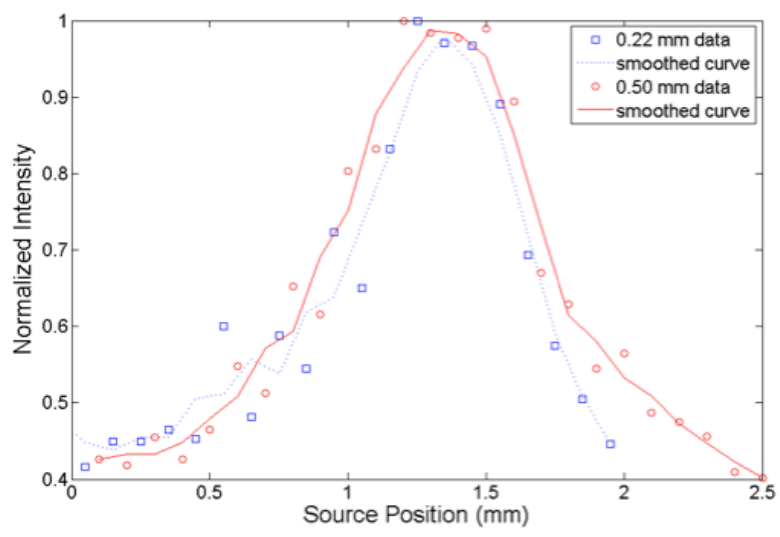

Figure 7: The crystal response functions from the $0.22 \times 0.22 \times 20 \mathrm{~mm}^{3}$ LSO array (blue) and from the $0.50 \times 0.50 \times 20 \mathrm{~mm}^{3}$ LSO array

\section{OBJECTIVE 2: INTEGRATION OF HIGH RESOLUTION DETECTOR WITH MICROPET II SCANNER FOR ADAPTIVE ZOOM-IN SCANNING:}

Aim: To optimize one such high resolution, high sensitivity detector and adaptively integrate it into the existing microPET II small animal PET scanner as a "zoom-in" detector that provides higher spatial resolution and sensitivity in a limited region close to the detector face.

Development of Image Reconstruction Methods for Adaptive Imaging: We developed a maximum a posteriori (MAP) reconstruction algorithm for the zoom-in PET system using both the high-resolution data (insert-scanner coincidence) and low-resolution data (scanner-scanner coincidence). The system matrix models the exact geometry of the microPET II scanner which we used in the experiment. It includes the detector block structure, solid angle effect, and crystal penetration effect. The attenuation of the high-resolution detector on the low-resolution data is also included. We analyzed theoretical the resolution and noise properties of the MAP reconstruction for the zoom-in PET. Using the theoretical results, we derived expressions for fast evaluation of the detectability of a lesion in a zoom-in PET image. Through various approximations, we developed a method that can predict the lesion detectability from a lowcount scout scan for a given position of the high-resolution detector in the zoom-in PET system. We then used the result to guide the positioning of the high-resolution detector to achieve the highest detectability of a certain signal, performing the so called "adaptive imaging".

Monte Carlo Simulation: We performed Monte Carlo simulation to validate the theoretical calculations and also to study the improvement in resolution and lesion detectability offered by the zoom-in PET system. We found good agreement between Monte Carlo simulation results and theoretical predications for the point spread function, noise variance, and signal-to-noise ratio for lesion detection. Simulation results show that with a $250 \mu \mathrm{m}$ detector, the zoom-in PET can improve the lateral resolution (parallel to the face of the high-resolution detector) from 0.8 $\mathrm{mm}$ for the microPET II alone to about $0.3 \mathrm{~mm}$ in the targeted region near the high-resolution detector. The improvement in spatial resolution and sensitivity also translated into more accurate quantification of regional uptake. Simulation results demonstrated reduced bias at any 
given noise level in quantifying the mean uptake in regions of different sizes. The figure to the right shows a comparison between reconstructed images obtained by the microPET II scanner and zoom-in PET (computer simulation). The $3 \times 3$ hot spots in the coronal slice can be clearly resolved by the zoom-in PET, but not by the microPET II scanner. More details can be found in (Zhou and Qi, 2009).

Monte Carlo simulations also showed that the high-resolution adaptive PET imaging is feasible. We showed that the maximum SNR for lesion detection can be reliably predicted from a scout scan with as low as $25 \mathrm{k}$ events (in a 2D sinogram). The optimal position

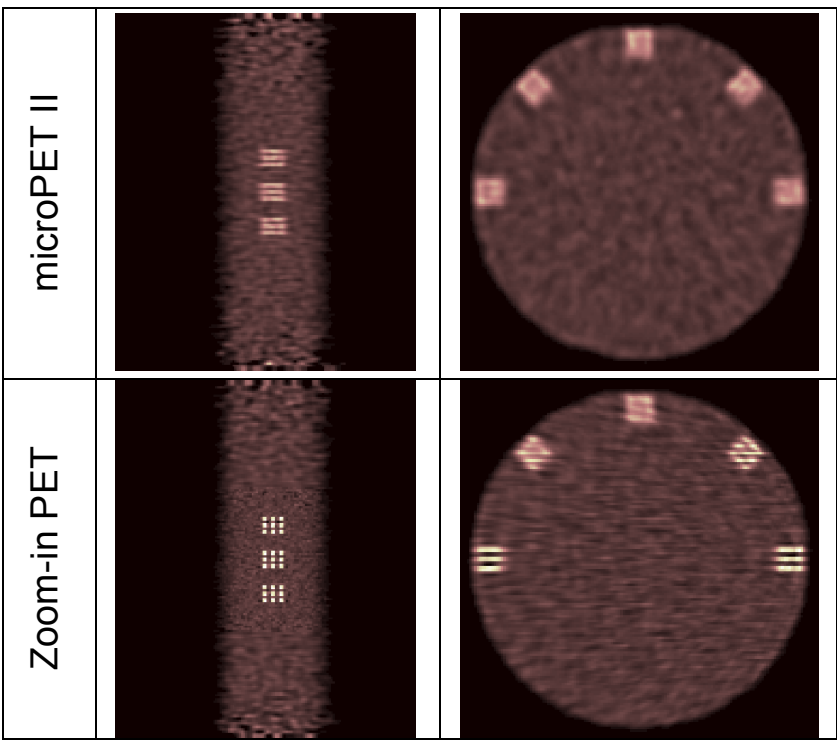
angle of the high-resolution detector can be clearly identified from the theoretical prediction and the result is confirmed by Monte Carlo simulation studies. It shows typically a well-positioned high-resolution detector can yield about 25\% improvement in SNR for detecting different type of signals over the microPET II scanner. Details can be found in (Zhou and Qi, 2011).

Experimental Characterization: We performed physical experiments to assess the resolution improvement by incorporating a high-resolution detector into the microPET II scanner. Because the microPET scanner electronics cannot handle dual-end readout for decoding DOI information, we did not use the $220 \mu \mathrm{m}$ DOI-capable detector as the insert in this experiment. Rather we used a high-resolution detector array that was made of a $14 \times 28$ array of $0.5 \times 0.5 \times 10 \mathrm{~mm}^{3}$ LSO elements and coupled it to two 64-channel photomultiplier tubes (PMTs) via tapered optical fiber bundles. The PMT signals are read out by the electronics in the microPET II scanner. In order to emulate measurements of DOI, the high-resolution detector was positioned sideways such that the long side of the crystal is parallel to the scanner axis. We use this experimental setup to investigate the spatial resolution improvement in transaxial planes. A $15 \mu \mathrm{Ci} \mathrm{Na}-22$ point source was positioned at various locations above the high-resolution detector. Images were reconstructed using the data measured by the microPET II scanner alone, and the microPET II
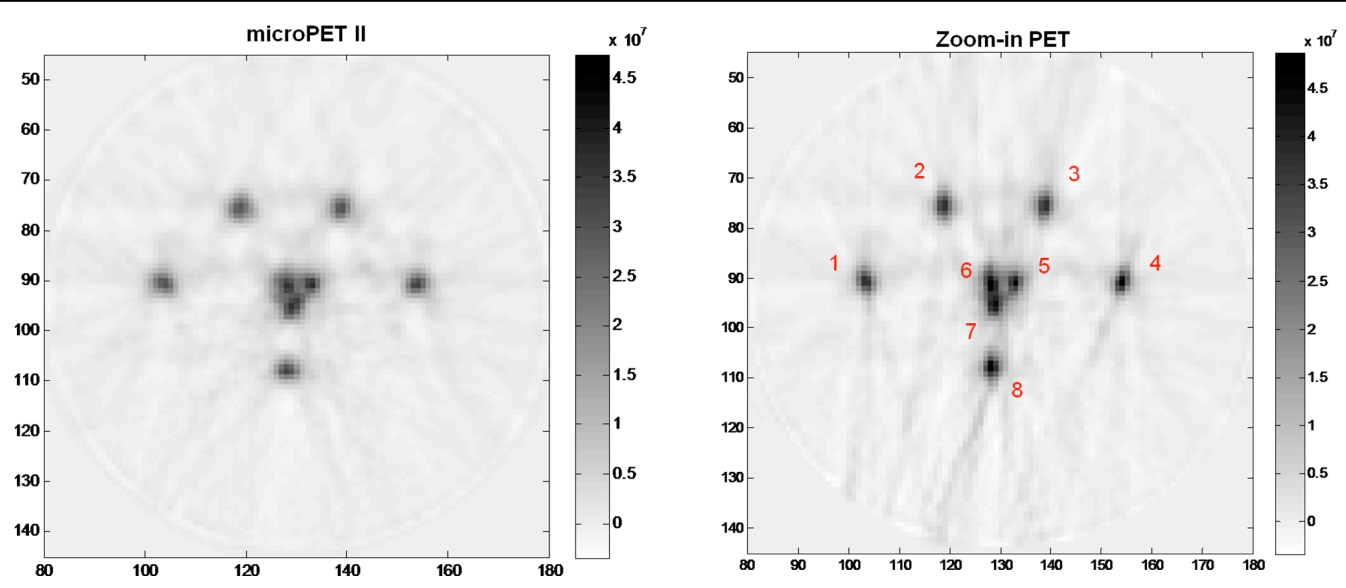

Point source images reconstructed from real measurements obtained by the microPET II (left) and the zoom-in PET (right). The image pixel size is $0.2 \mathrm{~mm} \times 0.2 \mathrm{~mm}$. 
data combined with the high-resolution detector data. Profiles taken through the reconstructed point sources show about $31 \%$ reduction in full-width-at-half-maximum (FWHM) along the direction parallel to the face of the high-resolution detector. Details of the experiment and results can be found in (Qi, et al, 2011).

\section{Journal Publications:}

1. St. James S, Yang $\mathrm{Y}, \mathrm{Wu} \mathrm{Y}$, Farrell R, Dokhale $\mathrm{P}$, Shah $\mathrm{K}$, Cherry SR. Experimental characterization and system simulations of depth of interaction PET detectors using 0.5 $\mathrm{mm}$ and $0.7 \mathrm{~mm}$ LSO arrays. Physics in Medicine and Biology 54: 4605-4619 (2009).

2. J Zhou and J Qi, "Theoretical analysis and simulation study of a high-resolution zoom-in PET system," Physics in Medicine and Biology, 54:5193-5208, 2009.

3. St. James S, Yang Y, Bowen SL, Qi JY, Cherry SR. Simulation study of spatial resolution and sensitivity for tapered depth of interaction PET detectors for small animal imaging. Physics in Medicine and Biology 2010; 55: N63-74.

4. J Zhou and J Qi, "Adaptive imaging for lesion detection using a zoom-in PET system," IEEE Transactions on Medical Imaging, 30:119-130, 2011.

5. J. Qi, Y. Yang, J. Zhou, Y. Wu, S. R. Cherry, "Experimental Assessment of Resolution Improvement of a Zoom-in PET," Physics in Medicine and Biology, 2011; 56: N165-174.

\section{Other Publications:}

1. Ph.D. Thesis: Sara St. James, University of California (2010)

2. J. Zhou and J. Qi, "High-resolution adaptive PET imaging," International Conference on Information Processing in Medical Imaging, LNCS 5636, 2009, pp. 26-37.

3. J. Zhou, S. St James, S. Cherry, and J. Qi, "Adaptive 3D PET Imaging using a HighResolution Detector," in International Meeting on Fully 3D Image Reconstruction, Beijing, China, 2009, pp. 215-218.

4. J. Zhou and J. Qi, "Efficient System Modeling of a High-Resolution Zoom-in PET Scanner," IEEE Medical Imaging Conference, Knoxville, TN, 2010, pp. 3501-3505.

5. J. Zhou and J. Qi, "Fast iterative image reconstruction using sparse matrix factorization with GPU acceleration," SPIE Medical Imaging Conference, Orlando, FL, 2011. Proc. SPIE 7961, 79610K (2011); doi:10.1117/12.878799 (7 pages) 